\title{
Effects of beam-tube roughness on x-ray free electron laser performance
}

\author{
Gennady Stupakov \\ Stanford Linear Accelerator Center, Stanford University, P.O. Box 4349, Stanford, California 94309 \\ Ruth Ellen Thomson \\ National Institute of Standards and Technology, Boulder, Colorado 80303
}

Dieter Walz

Stanford Linear Accelerator Center, Stanford University, P.O. Box 4349, Stanford, California 94309

Roger Carr

Stanford Linear Accelerator Center, Stanford University, P.O. Box 4349, Stanford, California 94309

(Received 5 April 1999; published 23 June 1999)

\begin{abstract}
In an x-ray free electron laser like the Linear Coherent Light Source being designed at SLAC, electron bunches about $70 \mu \mathrm{m}$ FWHM long are sent into a beam tube only $5 \mathrm{~mm}$ in internal diameter and more than $100 \mathrm{~m}$ in length. Because of the surface roughness of the beam tube, wakefields can be generated that catch up to the bunch and interact with it, causing energy spread and emittance growth. The strength of this effect depends on the details of the roughness of the surface. We present here a study in which the roughness of the beam tube was measured and the longitudinal impedance of the tube was calculated. Our result shows that a commercially available beam tube can be made smooth enough so the resulting wakefield effects are within the tolerance determined by the requirement that the induced relative energy spread of the beam be less than $5 \times 10^{-4}$. [S1098-4402(99)00050-6]

PACS numbers: 41.60.Cr, 41.75.Ht, 41.85.Ja
\end{abstract}

\section{INTRODUCTION}

At SLAC and DESY, x-ray free electron lasers are being designed to produce very high brightness $\mathrm{x}$-ray beams at $0.15 \mathrm{~nm}$ wavelengths and below. A linear accelerator will be used to deliver $5-15 \mathrm{GeV}$ or greater energy electron pulses, which enter undulators that may be more than $100 \mathrm{~m}$ long. If permanent magnets are used for the undulator, the allowable internal diameter of the beam tube is about $5 \mathrm{~mm}$. The undulator accepts a bunch of electrons and causes it to microbunch into transversely flat disks of electrons separated by the x-ray wavelength. The normalized emittance of the electron bunch should be of the order of $2 \pi \mathrm{mm}-\mathrm{mrad}$, and the peak current will be of the order of $3 \mathrm{kA}$. The transverse rms beam size is about only $30 \mu \mathrm{m}$, and its relative energy spread is of the order of $0.1 \%$.

During its passage through the undulator, the electron beam emits both coherent and incoherent radiation, and the intensity of the coherent radiation increases exponentially until it reaches saturation. It is essential that the emittance and energy spread of the beam not increase significantly, or the gain will decrease. In a previous study of the Linear Coherent Light Source (LCLS) [1], several wakefield effects were identified, and it was shown that the largest concern is the energy spread of the beam generated by the longitudinal impedance of the roughness on the inside surface of the beam tube. The emittance growth of the beam due to the roughness transverse wakefield imposes looser tolerances than the longitudinal effect. This impedance is generated by scattering of the beam field on the disturbances of the conducting surface of the tube. The effect of the surface resistance turns out to be less important so that, in the study of the roughness impedance, the surface can be considered as perfectly conducting.

Several models were recently developed for the calculation of the impedance of a perfectly conducting rough surface [2-5]. The result of Refs. [4,5], in the limit when the typical size of the roughness is much smaller than the length of the bunch, predicts an inductive longitudinal impedance

$$
Z(\omega)=-i L \omega
$$

where $Z$ is the impedance and $L$ is the inductance per unit length of the tube. In another model developed in Ref. [3], the impedance has a more complicated dependence versus frequency that includes a resistive part as well. As was shown in [6], in the limit when the bunch length is large compared to the size of the roughness, the resistive part of the impedance becomes small and, within a numerical factor, all three models agree with each other.

Assuming a Gaussian bunch of rms length $\sigma_{z}$, it is easy to find the energy spread of the beam at the end of the undulator generated by the impedance (1) (see, e.g., Ref. [1]):

$$
\delta E_{\mathrm{rms}}=\frac{N e^{2} L \mathcal{L} c^{2}}{3^{3 / 4} 2^{1 / 2} \pi^{1 / 2} \sigma_{z}^{2}},
$$

where $N e$ is the bunch charge and $\mathcal{L}$ is the length of the undulator. Inserting the parameters from Ref. [1] into 
Eq. (2), $\mathcal{L}=112 \mathrm{~m}, N e=1 \mathrm{nC}$, and $\sigma_{z}=20 \mu \mathrm{m}$, and assuming the final beam energy $E=14.3 \mathrm{GeV}$, one finds that the requirement of having the relative energy spread $\delta E_{\mathrm{rms}} / E$ increase less than $5 \times 10^{-4}$ gives the following tolerance for $L$ :

$$
L<1.6 \mathrm{pH} / \mathrm{m} \text {. }
$$

In this paper we report the results of measurements of the surface roughness of a tube similar to the one that will be used for the fabrication of the undulator beam tube. Based on these measurements, we calculate the impedance of the surface and compare it with the tolerance given by Eq. (3).

\section{MEASUREMENTS AND OBSERVATIONS}

In the LCLS, we plan to use round stainless steel beam tubes, internally plated with copper in order to reduce the resistive wall impedance. Ordinary stainless steel tubing has an internal surface roughness of about $R_{a}=1000 \mathrm{~nm}$, where $R_{a}$ is the arithmetic average of roughness feature sizes.

For this investigation, we procured high quality Type 316-L stainless steel tubing from the VALEX Corporation with an outer diameter of $6.35 \mathrm{~mm}$ and a wall thickness of $0.89 \mathrm{~mm}$. The order called for their best commercial finish, A5, corresponding to $R_{a}=125 \mathrm{~nm}$. The samples to be analyzed were cut from this tubing using an electrical discharge wire cutting process in order to eliminate damage from mechanical processing. The samples were subsequently cleaned chemically to remove particles adhering to the surface from the cutting process, which uses a brass wire.

To obtain good topographic maps of the beam tube surface, we examined these samples with an atomic force microscope (AFM). The AFM used employs the laser beam deflection technique to measure the displacement of the end of a silicon cantilever as it is rastered across the sample surface. The cantilevers we used were fabricated of single crystal silicon with the dimensions of $126 \mu \mathrm{m} \times$ $3.5 \mu \mathrm{m} \times 28 \mu \mathrm{m}$. These tips had resonant frequencies between 245 and $255 \mathrm{kHz}$. On the end of the lower surface of the cantilever is a pyramidal tip with an end radius of $10-50 \mathrm{~nm}$. The horizontal and vertical position of the tip is controlled by a piezoelectric scanner. Because we were primarily interested in length scales between 1 and $100 \mu \mathrm{m}$ we used a large stage AFM with the maximum lateral scan range of $108 \mu \mathrm{m}$. The AFM had a height range of only $5 \mu \mathrm{m}$, so careful positioning of the sample was required for the large scans since just the curvature of the tube could produce a height change of nearly $3 \mu \mathrm{m}$ if the scan was started at the lowest point of the tube. If the sample was incorrectly positioned and the scan was begun part way up the side of the tube, the height change of the sample over the $100 \mu \mathrm{m}$ scan would exceed the height range of the AFM, producing spurious data.
We obtained surface plots of the beam tube in intermittent contact ("tapping") mode. In this mode the AFM tip is oscillated at its resonant frequency by a small piezoelement located immediately behind the cantilever chip. As the tip approaches the surface the amplitude of this oscillation is reduced as the tip begins to contact the surface at the extreme end of its swing. The AFM uses this oscillation amplitude as the input to the feedback circuit that controls the position of the tip. We found that this mode of operation resulted in the surface maps with the best resolution and fewest artifacts.

We obtained AFM images $100 \mathrm{~nm} \times 100 \mathrm{~nm}$, $300 \mathrm{~nm} \times 300 \mathrm{~nm}, \quad 1 \mu \mathrm{m} \times 1 \mu \mathrm{m}, \quad 3 \mu \mathrm{m} \times 3 \mu \mathrm{m}$, $10 \mu \mathrm{m} \times 10 \mu \mathrm{m}, \quad 30 \mu \mathrm{m} \times 30 \mu \mathrm{m}$, and $100 \mu \mathrm{m} \times$ $100 \mu \mathrm{m}$. The smaller images were taken at faster scan rates $(1-3 \mathrm{~Hz})$ to reduce the effect of sample drift whereas the largest images required very slow scan rates $(0.1-0.2 \mathrm{~Hz})$ in order to accurately track the sample with the tip over such a large distance. It is essential to process the AFM images to flatten them and thus remove the effects of drift.

The small scale AFM images showed a relatively smooth surface as can be seen in Fig. 1a. A typical surface roughness for an image of $1 \mu \mathrm{m}$ or less was less than $1 \mathrm{~nm}$. Larger images revealed two interesting features. The most striking surface feature was the presence of nearly parallel grooves or striations that covered the entire sample surface. These grooves were typically about $1-5 \mu \mathrm{m}$ across and less than $100 \mathrm{~nm}$ deep and aligned with the axis of the beam tube. Two examples of images clearly showing these grooves are shown in Figs. 1f and 1g. Although not useful for the quantization of these grooves, optical microscope images show these grooves very clearly as seen in Fig. 2 .

The other outstanding feature of the surface was the presence of holes in the surface. Because of the finite width of the AFM tip it is not possible to determine if the AFM images reflect the true depth of these holes. They ranged from 0.2 to $7.5 \mu \mathrm{m}$ across and were from 4 to $250 \mathrm{~nm}$ deep, as measured by the AFM. These holes frequently appeared in groups as shown in Fig. 2. Most groups were aligned in a row in the same direction as the grooves mentioned earlier.

\section{THEORY}

For numerical analysis of the measurements, we used the theory developed in Ref. [4], which gives a simple expression for impedance of a rough surface in terms of the spectral function of the profile. This theory assumes a "small-angle" approximation, which, as is shown below, is well justified for the measured surface. The detailed derivation of the impedance of a rough surface can be found elsewhere [4]. Here we outline the main assumptions and present the final result of this model. 

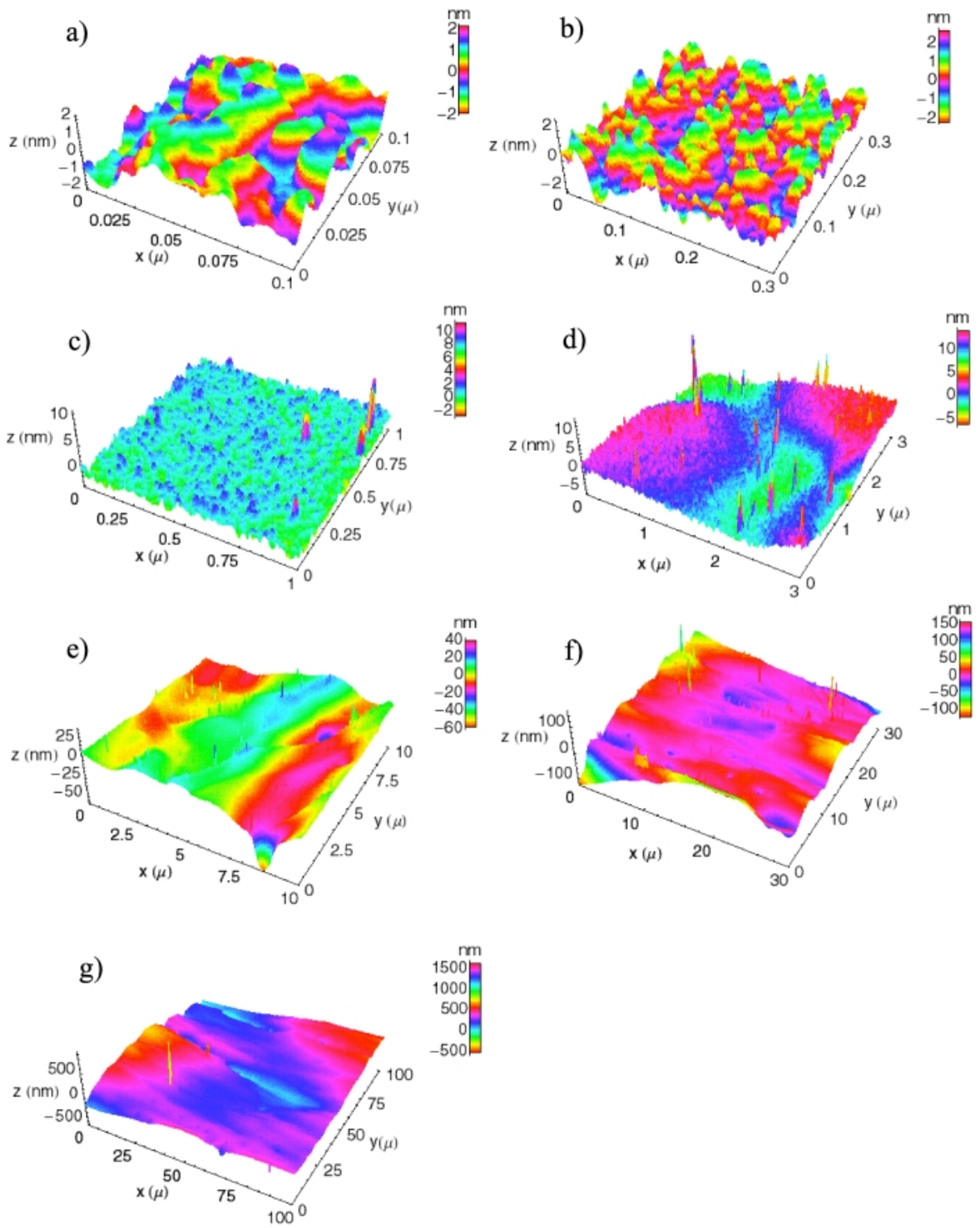

FIG. 1. (Color) AFM images ranging from $100 \mathrm{~nm} \times 100 \mathrm{~nm}$ to $100 \mu \mathrm{m} \times 100 \mu \mathrm{m}$. The color scale next to each image indicates the height of the surface.

The approach is based on the assumption that the angle between the normal to the rough surface and the radial direction is small compared to unity. If we assume that the rough surface is given by the equation $y=h(x, z)$, where $x, y$, and $z$ are the Cartesian coordinates and $h$ is the local height of the surface, than the small-angle approximation means that

$$
|\nabla h| \ll 1 .
$$

In addition to Eq. (4), we also require that the height of the bumps and their characteristic width $g$ be small compared to the radius of the tube $b$,

$$
g,|h| \ll b .
$$

We also assume that the characteristic frequency of interest $\omega \sim c / \sigma_{z}$, where $\sigma_{z}$ is the rms bunch length, is small compared to $c / g$,

$$
\omega \ll c / g \text {. }
$$

This also means that the size of the bumps $g$ is smaller than the bunch length $\sigma_{z}$.

Using approximations (4)-(6), one can show that inductance of a small bump of arbitrary shape $h_{0}(x, z)$ sitting on the surface of a round beam tube is

$$
L_{0}=\frac{Z_{0}}{c b^{2}} \int_{-\infty}^{\infty} \frac{\kappa_{z}^{2}\left|\hat{h}_{0}\left(\kappa_{z}, \kappa_{x}\right)\right|^{2}}{\sqrt{\kappa_{x}^{2}+\kappa_{z}^{2}}} d \kappa_{z} d \kappa_{x},
$$

where $\hat{h}_{0}$ is a two dimensional Fourier transform of the bump shape,

$$
\hat{h}_{0}\left(\kappa_{z}, \kappa_{x}\right)=\frac{1}{(2 \pi)^{2}} \int_{-\infty}^{\infty} \int_{-\infty}^{\infty} h_{0}(x, z) e^{-i \kappa_{z} z-i \kappa_{x} x} d z d x,
$$




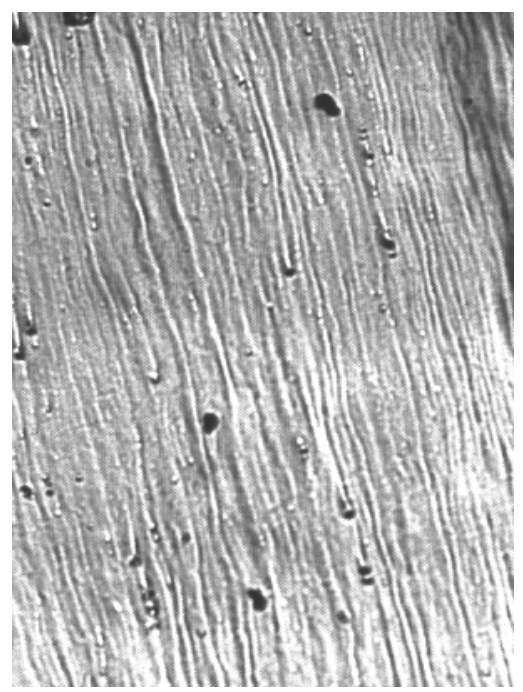

FIG. 2. Optical image of a surface. The vertical size of the image is $500 \mu \mathrm{m}$, and the horizontal size is $370 \mu \mathrm{m}$.

with the $z$ axis directed along the tube axis and the $x$ axis locally directed along the azimuthal coordinate $\theta$ in the cylindrical coordinate system associated with the tube. We note that, due to the assumed smallness of the surface features, we can use the local Cartesian coordinate system $x, y$, and $z$ in Eqs. (7) and (8) instead of the global cylindrical coordinate system $\theta, r$, and $z$.

To describe a rough surface with a random profile, we assume that $h(x, y)$ is a random function with zero average, $\langle h(x, z)\rangle=0$. Statistical properties of such a surface are characterized by the correlation function $K(x, y)$,

$$
K\left(x-x^{\prime}, z-z^{\prime}\right)=\left\langle h\left(x^{\prime}, z^{\prime}\right) h(x, z)\right\rangle,
$$

where the angular brackets denote averaging over possible realizations of $h(x, z)$. Equation (9) implies that statistical properties of $h(x, z)$ do not depend on the position of the surface. An important statistical characteristic of the roughness is the spectral density (or spectrum) $R\left(\kappa_{z}, \kappa_{x}\right)$, defined as a Fourier transform of the correlation function,

$$
R\left(\kappa_{x}, \kappa_{z}\right)=\frac{1}{(2 \pi)^{2}} \int d x d z K(x, z) e^{-i \kappa_{x} x-i \kappa_{z} z} .
$$

If the surface is statistically isotropic (all directions in the $x-y$ plane are statistically equivalent), the spectrum $R$ depends only on the absolute value $\kappa$ of the vector $\left(\kappa_{x}, \kappa_{z}\right)$, $\kappa=\sqrt{\kappa_{x}^{2}+\kappa_{z}^{2}}, R=R(\kappa)$.

The main result of Ref. [4] is that the longitudinal impedance (per unit length) of a circular tube of radius $b$ with a rough perfectly conducting surface characterized by the spectral function $R\left(\kappa_{x}, \kappa_{z}\right)$ in the frequency range limited by the condition (6) is given by Eq. (1) with the inductance equal to

$$
L=\frac{Z_{0}}{2 \pi c b} \int d \kappa_{z} d \kappa_{x} R\left(\kappa_{x}, \kappa_{z}\right) \frac{\kappa_{z}^{2}}{\kappa} .
$$

The presence of the factor $\kappa_{z}^{2}$ in the integrand of Eq. (11) means that the contributions to $L$ of roughness in the longitudinal $(z)$ and the azimuthal $(x)$ directions are different. For example, bellow-type variations on the surface have spectral components with $\kappa_{z} \neq 0$ and $\kappa_{x}=$ 0 and result in nonvanishing $L$. On the other hand, grooves of all sizes in the longitudinal direction, as described in the previous section, generate a spectrum with $\kappa_{x} \neq 0$ and $\kappa_{z}=0$ and according to Eq. (11) do not contribute to $L$.

\section{ANALYSIS}

Because of the limited number of measurements, the averaging over a representative statistical ensemble in Eq. (9) was not possible in our study. Instead, for calculation of the impedance, we used Eqs. (7) and (8) where the function $h_{0}(x, z)$ was the measured profile for a given sample. To take into account the small area of a sample, we multiplied Eq. (7) by $2 \pi b / S_{\text {sample }}$ equal to the number of samples that would fit the surface of a unit length of the tube,

$$
L=\frac{2 \pi Z_{0}}{b c S_{\text {sample }}} \int \frac{\kappa_{z}^{2}\left|\hat{h}_{0}\left(\kappa_{z}, \kappa_{x}\right)\right|^{2}}{\sqrt{\kappa_{x}^{2}+\kappa_{z}^{2}}} d \kappa_{z} d \kappa_{x} .
$$

This method of calculation assumes that the surface is composed of many pieces of size $S_{\text {sample, and the }}$ impedance of the whole surface is given by the sum of the impedances of each piece. Such assumption is approximately correct if the typical size of the roughness that contributes to the inductance is much smaller than the sample size. In the opposite limit, for very small samples, the superposition rule is not valid, and we should see that the impedance calculated using this approach varies with the size of the sample. Of course, one should also keep in mind that, due to random fluctuations of the profile from one sample to another, there will be associated with it variation of the impedance.

It follows from Eq. (12) that the product $L b$ does not depend on the tube radius $b$ and can be considered as a characteristic parameter of the surface microstructure. To calculate $L b$, we performed numerical Fourier transformation of the two-dimensional data arrays representing the surface $h_{0}(x, z)$. One such spectrum, for the largest sample of $100 \mu \mathrm{m} \times 100 \mu \mathrm{m}$, is shown in Fig. 3. It can be seen that the spectrum is localized at small values of the wave numbers $\kappa$, roughly corresponding to the inverse size of the sample. Those small values of $\kappa$ make a dominant contribution to the integral in Eq. (7).

Another important feature of the roughness can be deduced from Fig. 1. Comparing the vertical and horizontal scales on each image, we see that they typically differ by an order of magnitude, that is the typical height of the roughness is much smaller than the characteristic size of the bumps. This observation justifies the use of Eq. (7) that was derived using the small-angle approximation model in Ref. [4]. Unfortunately, the other condition of applicability of Eq. (7), given by Eq. (6), is only 


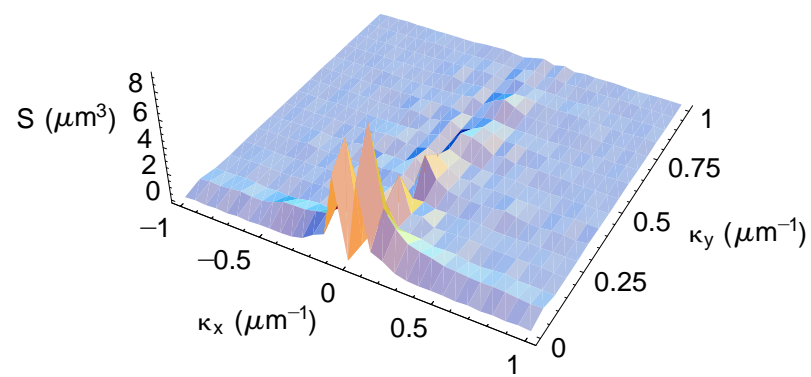

FIG. 3. (Color) Spectrum $S=\left|\hat{h}_{0}\left(\kappa_{x}, \kappa_{y}\right)\right|$ for the $100 \mu \mathrm{m} \times$ $100 \mu \mathrm{m}$ sample.

marginally satisfied for our calculations. This condition requires that the transverse dimension of the bumps, which for a continuous spectrum can be estimated as a typical inverse wavelength in the spectrum, $g \approx \pi / \kappa$, be smaller than the beam length. From Fig. 3 we conclude that $\kappa \approx 0.1 \mu \mathrm{m}^{-1}$, and hence $g \approx 30 \mu \mathrm{m}$, which is comparable to the rms bunch length of $20 \mu \mathrm{m}$.

After calculation of the spectra for each sample, the integral in Eq. (12) was estimated numerically, and the corresponding values of the product $L b$ are given in Table I together with the rms height $h_{\mathrm{rms}}$ of the roughness for each sample. Since inductance $L$ depends on the sample orientation relative to the direction of the beam propagation ( $z$ axis), we calculated $L b$ for two orthogonal orientations of the samples indicated by indices 1 and 2. In the first case, the $z$ axis of the sample was directed along the tube axis; in the second case, it was assumed that the $x$ axis of the sample goes along the tube axis.

From Table I we can see that $h_{\text {rms }}$ increases for larger samples from about $1 \mathrm{~nm}$ for the $0.1 \mu \mathrm{m} \times 0.1 \mu \mathrm{m}$ sample to about $100 \mathrm{~nm}$ for the $100 \mu \mathrm{m} \times 100 \mu \mathrm{m}$ sample. With the increase of the roughness, the value of $L b$ also grows reaching the maximum value of about $2.5 \times 10^{-4} \mathrm{pH}$.

As we see from Table I, the maximum inductance corresponds to the largest sample in the table. This fact agrees with the observation that the roughness increases with the size of the sample. In order to obtain a better statistical representation for the largest scales available for measurements with AFM, we carried out several more measurements for samples of the size of about $100 \mu \mathrm{m}$. Those measurements are presented in Table II. We see

TABLE I. Product $L b$ for different samples.

\begin{tabular}{cccc}
\hline \hline Sample size & $h_{\mathrm{rms}}(\mathrm{nm})$ & $(L b)_{1}(\mathrm{pH})$ & $(L b)_{2}(\mathrm{pH})$ \\
\hline $0.1 \mu \mathrm{m} \times 0.1 \mu \mathrm{m}$ & 0.8 & $1.9 \times 10^{-5}$ & $2.5 \times 10^{-5}$ \\
$0.3 \mu \mathrm{m} \times 0.3 \mu \mathrm{m}$ & 0.7 & $1.0 \times 10^{-5}$ & $1.0 \times 10^{-5}$ \\
$1 \mu \mathrm{m} \times 1 \mu \mathrm{m}$ & 0.8 & $9.8 \times 10^{-6}$ & $8.8 \times 10^{-6}$ \\
$3 \mu \mathrm{m} \times 3 \mu \mathrm{m}$ & 2.0 & $1.1 \times 10^{-5}$ & $8.8 \times 10^{-6}$ \\
$10 \mu \mathrm{m} \times 10 \mu \mathrm{m}$ & 9.5 & $2.7 \times 10^{-5}$ & $3.5 \times 10^{-5}$ \\
$30 \mu \mathrm{m} \times 30 \mu \mathrm{m}$ & 17.6 & $8.6 \times 10^{-5}$ & $3.5 \times 10^{-5}$ \\
$100 \mu \mathrm{m} \times 100 \mu \mathrm{m}$ & 92.1 & $2.5 \times 10^{-4}$ & $2.6 \times 10^{-4}$ \\
\hline \hline
\end{tabular}

TABLE II. Product $L b$ for larger samples.

\begin{tabular}{cccc}
\hline \hline Sample size & $h_{\mathrm{rms}}(\mathrm{nm})$ & $(L b)_{1}(\mathrm{pH})$ & $(L b)_{2}(\mathrm{pH})$ \\
\hline $108 \mu \mathrm{m} \times 108 \mu \mathrm{m}$ & 98 & $4.1 \times 10^{-4}$ & $2.8 \times 10^{-4}$ \\
$85 \mu \mathrm{m} \times 85 \mu \mathrm{m}$ & 84 & $4.0 \times 10^{-4}$ & $3.9 \times 10^{-4}$ \\
$65 \mu \mathrm{m} \times 65 \mu \mathrm{m}$ & 109 & $3.5 \times 10^{-4}$ & $1.9 \times 10^{-3}$ \\
$104 \mu \mathrm{m} \times 104 \mu \mathrm{m}$ & 186 & $7.0 \times 10^{-4}$ & $2.7 \times 10^{-4}$ \\
\hline \hline
\end{tabular}

that they typically have about the same value of $h_{\mathrm{rms}}$ and similar inductance as the largest sample in Table I (except for one value in the third row).

We calculated the average value of $L b$ for five samples with the size larger than $65 \mu \mathrm{m}$. For two different orientations of the samples, they are $(\overline{L b})_{1}=4.2 \times$ $10^{-4}$ and $(\overline{L b})_{2}=6.2 \times 10^{-4}$. Those values should be compared with the tolerance given by Eq. (3), which for $b=2.5 \mathrm{~mm}$ radius becomes $(L b)_{\text {tol }}=4 \times 10^{-3} \mathrm{pH}$. As we see, the measured values are several times smaller than the tolerance.

To verify that scales larger than $100 \mu \mathrm{m}$ have approximately the same level of roughness, we also measured the surface profile on the length up to $2 \mathrm{~mm}$ using a profilometer. One such profile is shown in Fig. 4. As can be seen, typically, the variation of the surface height is within several hundreds nanometers, except for a small number of zones where it jumps to about $1 \mu \mathrm{m}$. This picture qualitatively confirms that the samples in Table II can be considered as typical representatives of the surface roughness on the scale of $0.1 \mathrm{~mm}$.

\section{SUMMARY}

We measured the roughness of a stainless steel beam tube of a type similar to the one which will be used for the undulator tube. The measurements show that the roughness increases with the size of the sample up to the largest size of $100 \mu \mathrm{m}$. An important feature of the roughness found is that the typical height of the bumps is much smaller than their size in horizontal direction. This feature allowed us to use the impedance theory developed in Ref. [4] in the small-angle approximation.

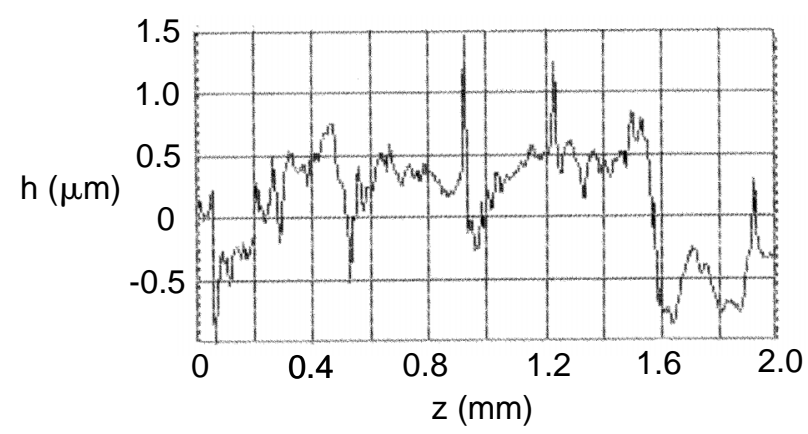

FIG. 4. Surface profile along the tube axis measured by a profilometer. 
Based on this theory we calculated the inductance for different sizes of samples and showed that the expected impedance of the tube is several times smaller than the tolerance given by the requirement of having the induced energy spread increase due to an impedance less than $0.05 \%$. The calculated impedance turns out to be smaller than previously estimated in Ref. [1] (for a given average height of the bumps) due to the small aspect ratio of height to width for the roughness. A simple model used in Ref. [1] assumes $h \sim g$, thereby overestimating the impedance and imposing more tight tolerances in terms of the rms height.

Although our results predict an impedance that is well below the tolerable level for LCLS, one has to keep in mind several important assumptions made in the theory. First, it assumes that the length of the bunch is much larger than the horizontal size of the roughness. This assumption is only marginally satisfied for the LCLS beam with $20 \mu \mathrm{m}$ rms length. Another important effect that lies beyond the scope of our model is the possibility of the existence of a single synchronous mode in a rough tube predicted in Ref. [3]. Consideration of the effect of this mode on the LCLS beam is given in Ref. [6]. Finally, note that we used a Gaussian profile for the beam charge density, although the LCLS bunch is expected to have a more flat distribution.

For these reasons and to introduce an element of conservatism into the LCLS vacuum chamber design, there is an effort under way to improve on the surface roughness of commercially available high quality stainless steel tubing using additional electropolishing. These high quality internal surfaces will then be coated with copper to minimize the resistive contribution to the wakefields. This process will be followed by additional electropolishing to minimize the surface roughness.

\section{ACKNOWLEDGMENTS}

G. S, R. C., and D. W. were supported in this work by the U.S. Department of Energy, Office of Basic Energy Sciences, under Contract No. DE-AC03-76SF00515. Part of this work (R.E.T.) was contributed by the National Institute of Standards and Technology and is not subject to copyright.

[1] The LCLS Design Study Group, SLAC Report No. SLACR-521 (unpublished).

[2] K. L.F. Bane, C. K. Ng, and A. W. Chao, SLAC Report No. SLAC-PUB-7514 (unpublished).

[3] A. Novokhatski and A. Mosnier, in Proceedings of the 1997 Particle Accelerator Conference, Vancouver, B.C., Canada, 1997 (IEEE, Piscataway, NJ, 1997), pp. 16611663.

[4] G. V. Stupakov, Phys. Rev. ST Accel. Beams 1, 064401 (1998).

[5] K. L. F. Bane and G. V. Stupakov, International Computational Accelerator Physics Conference (ICAP 98), Monterey, CA, 1998 (unpublished), SLAC Technical Report No. SLAC-PUB-8023.

[6] K. Bane and A. Novokhatskii, SLAC Technical Report No. SLAC-AP-117 (unpublished). 